\section{Human auditory-evoked response: Specific effects of signal strength and performance criterion*}

\author{
DENNIS A. BENSON† \\ Department of Psychology \\ and \\ D. C. TEAS \\ Communication Sciences Laboratory, Department of Speech \\ University of Florida, Gainesville, Florida 32601
}

Computer-averaged auditory-evoked responses were recorded from the scalp of seven human Ss in order to study the effects of both low-signal levels and different performance criteria upon specific components of the waveform. Noise bursts of $-2,0,+2$, or $+6 \mathrm{~dB}$ sensation level (SL) were presented contingent upon the S's correct bisection of a temporal interval as the degree of accuracy required from him was systematically varied. Analyses of variance indicated that a positive peak in the waveform at a latency of approximately $300 \mathrm{msec}$ increased in amplitude as the stringency of the performance criterion increased but showed no change as signal strength varied. The negative peak at approximately $125 \mathrm{msec}$ latency increased with increasing signal strength but remained constant as the stringency of the performance criterion was varied.

The waveform of the averaged evoked response (AER) recorded from the scalp of human $\mathrm{Ss}$ can be altered by modifying the psychological context in which the responses are recorded (Haider, Spong, \& Lindsley, 1964; Wilkinson, Morlock, \& Williams, 1966; Ritter \& Vaughan, 1969; Sheatz \& Chapman, 1969). Most studies have simply stated that an increase in attention to the stimulus results in an increase in the amplitude of the AER (Davis, 1964; Satterfield, 1965; Gross, Begleiter, Tobin, \& Kissin, 1965; Mast \& Watson, 1968). Generally speaking, these experiments have used high-intensity stimulus values and have failed to specify exactly what components in the complex AER waveform increases with increasing attention.

The auditory stimulus intensities in such experiments have commonly been in the range of 70-90 dB SL, e.g., $70 \mathrm{~dB}$ SL (Davis, 1964), $75 \mathrm{~dB} \mathrm{SL}$ (Sheatz \& Chapman, 1969), and 90 dB SL (Gross et al, 1965). Only Mast and Watson (1968) have utilized any form of an attention paradigm to record responses near behavioral thresholds. When their Ss were instructed to count the stimuli, Mast and Watson recorded a discernible AER down to $1 \mathrm{~dB}$ SL for the two Ss whose data were reported.

* This research was supported in part by Grant NS-07287 and Program Project No. NB-06459 from the Public Health Service, NIH. Mr. Benson is the recipient of an NSF traineeship. This manuscript is a revision of an MS thesis submitted in August 1970.

Address: Department of Psychology, University of Florida, Gainesville, Florida 32601 .
The conclusions of many studies that examine the AER as a function of attention are based on the overall or peak-to-peak amplitude of the AER waveform and neglect the changes in the individual positive and negative components (Satterfield, 1965; Gross et al, 1965; Ritter, Vaughan, \& Costa, 1968). In those studies, however, which have included measurements of specific components of the AER, investigators have found that different degrees of attention produce different effects upon the various positive and negative components of the AER (Garcia-Ausst, Bogacz, \& Vanzulli, 1964; Wilkinson et al, 1966; Wilkinson \& Morlock, 1966). Failure to specify the exact location of the enhancement in the waveform led Mast and Watson (1968) to conclude that the increase in the AER due to increased attention is greater at low stimulus intensities than at high stimulus intensities. Close examination of the waveforms presented in their paper indicates that the principal change in the waveform in their "counting condition" was the appearance of a late positive peak. In their figure, this peak had the same magnitude for both the 5- and 30-dB SL stimuli within the "counting condition." Since the negative component preceding the late positive component was considerably larger at $30 \mathrm{~dB}$ SL whether or not the stimulus was attended to, the peak-to-peak measurement does not reveal the consistency of the attentiondependent positive component for the two stimulus strengths.

Some physiological support for fractionating the AER into separate components comes from the work of Vaughan and Ritter (1970). These investigators recorded the AER to an acoustic input from a variety of locations on the scalp. They found that the AER waveform and the scalp distribution of AERs for periodically presented acoustic stimuli were substantially different from the waveforms and scalp distribution of AERs for infrequent aperiodic stimuli.

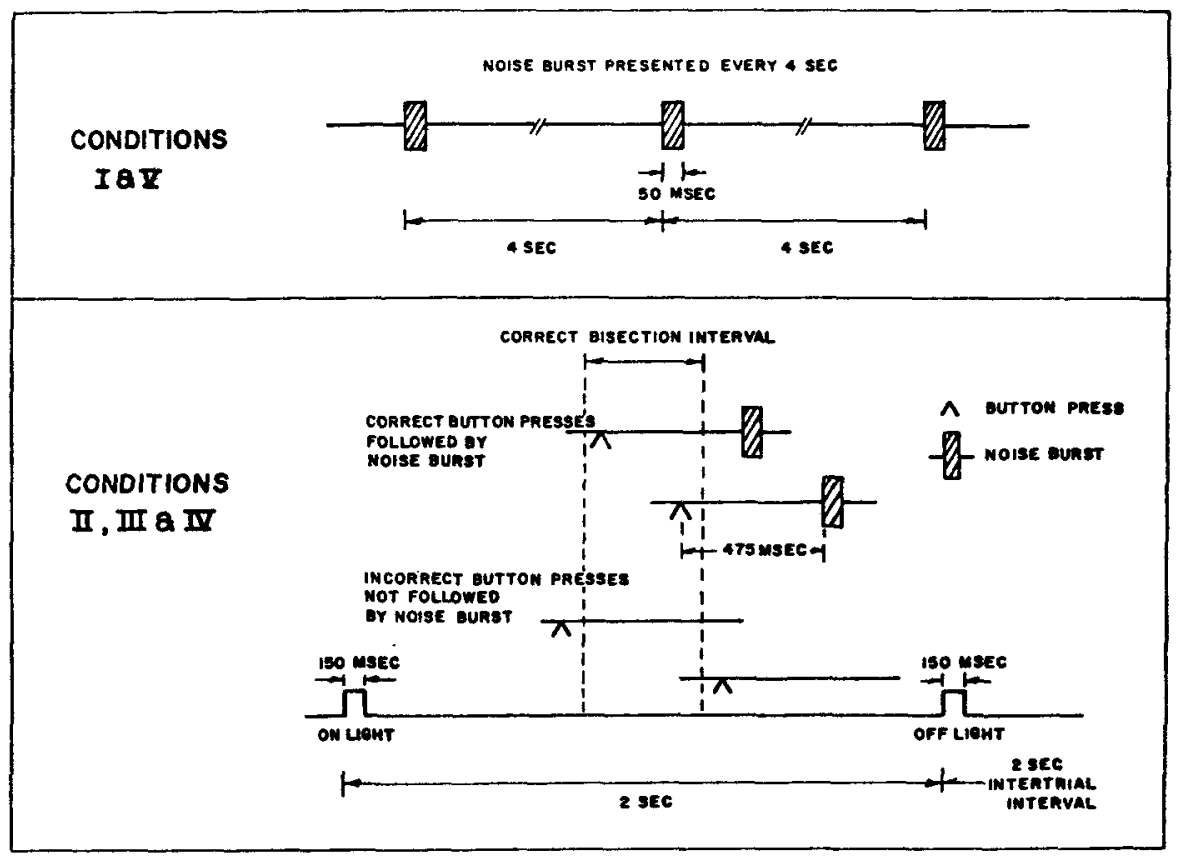

Fig. 1. Schematic diagram of the experimental procedure. 
Table 1

Mean Proportion of Correct Responses for the Experimental Condition: as a Function of Sequence of Conditions

\begin{tabular}{lcccc}
\multicolumn{1}{c}{$\begin{array}{c}\text { Sequence of } \\
\text { Conditions }\end{array}$} & N & $\begin{array}{c}\text { II } \\
\text { (800 Msec) }\end{array}$ & $\begin{array}{c}\text { III } \\
\text { (400 Msec) }\end{array}$ & $\begin{array}{c}\text { IV } \\
\text { (250 Msec) }\end{array}$ \\
\hline $\begin{array}{l}\text { Sequence 1: (II, III IV) } \\
\text { Sequence 2: (II, IV, III) }\end{array}$ & 4 & .950 & .830 & .613 \\
$\begin{array}{l}\text { Unweighted Mean } \\
\text { Proportion Correct }\end{array}$ & 3 & .900 & .663 & .545 \\
\hline
\end{tabular}

Their data suggest that different cortical sources account for the separate components that constitute the two different AER waveforms. No experiment has specifically investigated the effects of attention on low-intensity stimuli in terms of these specific components of the AER. The present experiment, therefore, was designed to analyze the simultaneous effects of physical and psychological variables on individual components of the AER to low-intensity stimuli.

\section{METHODS AND PROCEDURES} Stimuli

Stimulus strength was expressed re the S's threshold. Four stimuli were used: $-2,0,+2$, and $+6 \mathrm{~dB}$ SL. One stimulus was below threshold so that a change in AER threshold could be observed, should it occur; the narrow range of low-stimulus strengths allowed the $S$ to maintain a "set" for low-level acoustic signals. Threshold was approximated by a bracketing procedure, in which ascending and descending trials were alternated. The signals used for threshold determination were the same type of signals used in the experiment: 50 -msec noise bursts with a $0.1-\mathrm{msec}$ rise time. The signal strength at which the $S$ made two out of three correct detections of the signal was defined as 0 dB SL. Each S's threshold was checked three times throughout the experiment. When a change in threshold occurred, a corresponding change was made for the acoustic stimulus so that $0 \mathrm{~dB}$ SL would be maintained.

The four stimuli were presented in random order by automated equipment. The output of a wide-band noise generator (Grason-Stadler, Model $455 \mathrm{C}$ ) was fed to four separate electronic switches with relative gains corresponding to the four stimulus strengths. A random selection of one of the four switches was made for each trial by the automated programmer. The selected signal was then passed through an amplifier (J. B. Lansing, Model SE400S) and an attenuator (Hewlett-Packard, Model 350D) which allowed the $\mathrm{E}$ to set the value for
0 dB SL. Stimuli were delivered by headphone (Grason-Stadler, Model TDH-39) to the right ear only.

\section{Behavioral Constraints}

Superimposed on the random selection for each trial of one of the four stimulus strengths was a behavioral task. In order for a stimulus to be presented, the $S$ was required to bisect a 2-sec time interval. His bisection had to fall within a time zone located symmetrically about the 1 -sec point. These time zones were termed "correct intervals" (CIs) and were $\pm 400 \mathrm{msec}, \pm 200 \mathrm{msec}$, or $\pm 125 \mathrm{msec}$. Figure 1 schematizes the essential features of the five conditions that constituted an experimental session.

In Conditions I and V, Ss were instructed only to sit quietly with their eyes open. A noise burst, selected randomly from the four programmed levels of $-2,0,+2$, and +6 dB SL was presented once every $4 \mathrm{sec}$. The condition terminated when each level of the signal had been presented at least 12 times. Conditions $I$ and $V$ were always the first and last conditions, respectively, in each experimental session and served as controls.

In the experimental conditions (Conditions II, III, and IV), the S was required to perform the temporal bisection task. $A$ black box containing two signal lights spaced 12 in. apart was mounted on the wall at eye level approximately $5 \mathrm{ft}$ in front of the $S$. The left was designated the "on" light and the right light the "off" light. A brief flash from the "on" light was followed $2 \mathrm{sec}$ later by a flash from the "off" light. The S's task was to bisect the interval by pressing a microswitch at the estimated midpoint. The switch closure caused a deflection of a galvanometer mounted in the signal light box midway between the "on" and "off" lights. The galvanometer thus provided the $S$ with a fixation point as well as feedback of the time of his buttonpress in relation to the flashing of the "on" and "off" lights.

The $S$ was informed that his judgment would be considered "correct" if the buttonpress occurred within a certain temporal interval centered at the exact midpoint of the 2-sec interval. "Correct" judgments produced the selected noise burst 475 msec after the buttonpress. No noise burst occurred after incorrect judgments. The $\mathrm{S}$ was also told that the intensity of the noise burst was random and in no way related to his accuracy. To insure that the $S$ had understood the instructions, a few practice trials were given with a very wide CI, after which Condition II began. For Condition II, the CI was $\pm \mathbf{4 0 0}$ msec; thus, a buttonpress would have to occur within \pm 400 msec of the exact midpoint of the 2-sec interval in order to be considered correct. For Condition III, the CI was \pm 200 msec, and for Condition IV the CI was \pm 125 msec. A written record of the time ( \pm 1 msec) between the "on" light and the buttonpress for each trial was obtained from an electronic counter (Hewlett-Packard, Model 5214L).

Each condition consisted of as many trials as necessary to accumulate 12 correct responses at each of the four intensity levels. Before each condition began, the $S$ was given a few minutes to relax, informed of the relative stringency of that condition's CI, and encouraged to maximize his proportion of correct responses.

Seven undergraduates, four males and three females, served as Ss as part of a course requirement. The only criterion for selection was a hearing level within normal limits for the noise burst. The Ss were assigned randomly to two different sequences of experimental conditions. Four $\mathrm{Ss}$ were

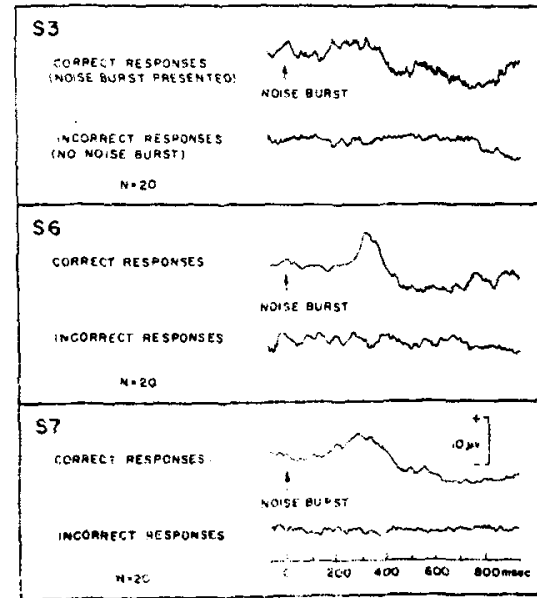

Fig. 2. Comparison of averaged responses when noise burst was presented (correct responses) with averaged activity when no auditory stimulus was presented (incorrect responses). 


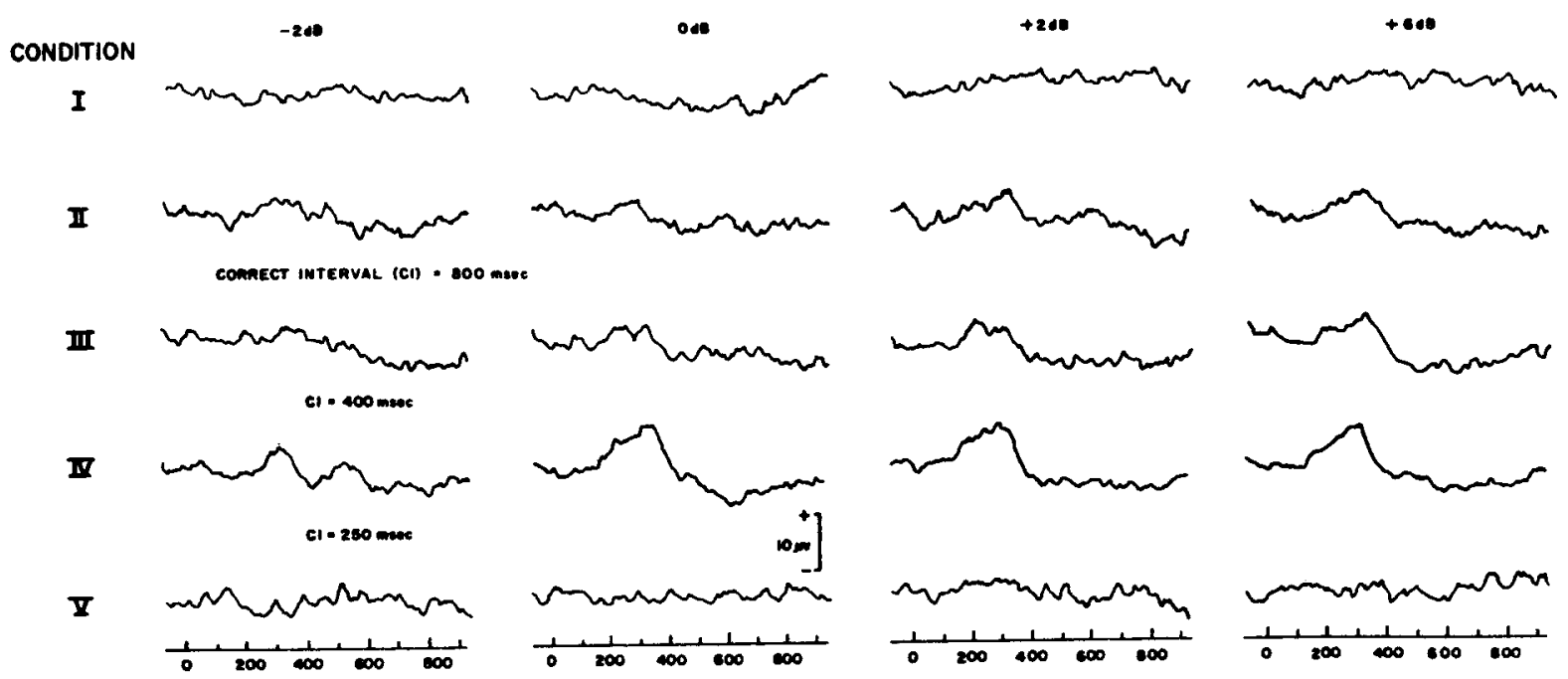

THE FROM STMMULUS ONSET (msec)

Fig. 3. AERs from one $S$ (S 7 ) for each of the 20 treatment combinations. Each AER is based on 12 individual responses.

run in the order of increasing stringency (Sequence 1): Conditions II (CI $= \pm 400 \mathrm{msec})$, III ( $\pm 200 \mathrm{msec}$ ), and IV ( $\pm 125 \mathrm{msec})$. Three Ss received Sequence 2: Conditions II, IV, and III. A complete reversal of sequences, i.e., Conditions IV, III, and II, was not attempted, since a pilot study showed that Ss could not perform the task if the condition with the most stringent CI, Condition IV, was presented first.

The complete experimental session lasted approximately $1 \mathrm{~h}$.

\section{Recording Procedures}

All the major events in the experimental paradigm, viz, the occurrence of the "on-off" lights, buttonpresses, and acoustic stimuli, as well as the S's EEG, were recorded on an FM tape recorder (Ampex, Model FR-100) for off-line analysis of the data.

Recordings of the EEG were made from a silver disk electrode attached with electrode paste to the vertex, $C_{Z}$ of the "10-20 system" (Jasper, 1958). The reference electrode was clipped to the left earlobe and a ground to the right earlobe. For two $\mathrm{Ss}$, an additional electrode was placed on the neck and referred to the left earlobe in order to detect any muscular artifacts. When no artifacts were observed in off-line processing for those Ss, the neck recordings were discontinued for subsequent Ss, and that channel was used to record "incorrect" buttonpresses. The electrodes were led to high-impedance probes (Grass, Model HIP-511B) and then to high-gain (20,000x) amplifiers (Grass P511) with a pass band of 0.3 to $100 \mathrm{~Hz}$. In off-line processing, the signal was further filtered by a $60-\mathrm{Hz}$ low-pass filter. The outputs of the amplifiers were monitored on an oscilloscope (Tektronix, Model RM561A) and led to both the FM tape recorder and the averaging computer, HAVOC. ${ }^{1}$ On-line monitoring of the AER was accomplished by computing averages of the evoked responses to 20 noise bursts of mixed intensity and displaying the average on HAVOC's oscilloscope. The primary data analysis was performed off-line to facilitate sorting of responses according to the different experimental categories. The off-line EEG was written out on a pen recorder (Sanborn, Model 150) as a check for artifacts in the tape-recorded signal. If a movement or other artifact occurred, that sample was eliminated from the average. The computer averages of the AERs were graphically recorded with an $X-Y$ plotter (Omnigraphic Corp., Model HR-195).

\section{Data Analysis}

In the off-line analysis of the AER separate averages corresponding to each stimulus intensity and condition were recovered from the tape for each S. For averaging, the sweep was triggered by the onset of "correct" buttonpresses. Since the acoustic stimulus followed the buttonpress by $475 \mathrm{msec}$, an estimate of the prestimulus baseline was present for each averaged response. All measurements were made from this prestimulus baseline (B), which was defined as a horizontal line passing through the average amplitude of the 75-msec period preceding a stimulus. Because the responses were of low amplitude, temporal criteria were used to define the location within the AERs at which amplitude measurements were made. The most negative point in the waveform between 100 and 150 msec was defined as $N_{1}$. The most positive point occurring between 275 and $325 \mathrm{msec}$ was defined as $P_{2}$. Measurements of $B-P_{2}$ and $N_{1}-P_{2}$ were taken for all conditions and signal strengths. The $\mathrm{N}_{1}-\mathrm{B}$ measure, however, was taken for all conditions only at the +2 and $+6-d B$ SL signal strengths, since no $N_{1}$ deflections were distinguishable from the baseline at -2 or 0 dB SL.

The waveforms that were used for measuring the above components represented a 1 -sec sampling of the EEG occurring from $75 \mathrm{msec}$ before to 925 msec after the onset of the noise burst. Preliminary comparisons of the first six AERs with the last six AERs from the same condition and signal strength for a given $S$ revealed no measurable differences. Therefore, all 12 responses were averaged together to yield a single AER with a higher signal-to-noise ratio than would have been possible with an average based only on 6 responses.

As a control procedure, the computer was triggered on "incorrect" responses (after which no acoustic stimulus occurred) in order to detect any possible electrical or muscular artifacts resulting from the buttonpress itself.

\section{RESULTS}

\section{Behavioral Date}

Records of the number of correct and incorrect judgments for each experimental condition were used to provide a quantitative estimate of the Ss' performance under each of the different CIs. Table 1 shows the 
proportion of correct judgments as a function of the size of the CI for the two groups receiving different sequences of the CIs. The proportion of correct judgments was used as a measure of performance. The number of correct judgments for each condition was relatively constant, since at least 12 correct responses were required at each of the four signal strengths in order to compute an AER. The proportions of correct judgments were transformed to arcsin scores. An analysis of variance (unweighted means solution) showed that the two groups did not significantly differ in their proportion of correct judgments $(F=3.85, \mathrm{df}=1,5)$. Therefore, the data from the two groups were combined for the subsequent analyses. The effect of different CIs was highly significant $(\mathrm{F}=19.72, \mathrm{df}=2,10)$. A multiple comparison of the mean proportion correct for each CI showed that all three mean differences were significant $(p<.05)$. Thus, it can be inferred that the three different CIs represented three ordered and distinct stages of difficulty for the $S$. Comparisons of the number of errors in the first half of each condition vs the last half using the Wilcoxon signed-ranks test (Siegel, 1956) showed no significant differences for any of the CIs. This comparison indicated that Ss maintained a similar level of performance throughout each CI.

\section{Electrophysiological Data}

Prior to analyzing the AERs to the acoustic stimuli, control averages were obtained for each $S$ to determine if there was any observable electrical activity not due to the acoustic stimulus itself. These control waveforms were triggered by buttonpresses occurring outside the $\mathrm{CI}$-hence, there was no presentation of the noise burst. Figure 2 compares averaged responses produced by noise bursts (correct buttonpresses) with averaged activity when no auditory stimulus was presented (incorrect buttonpresses). Since both traces were triggered by the buttonpresses, they cover similar periods of time within the experimental paradigm. The figure shows that the waveforms following incorrect buttonpresses were essentially flat, indicating that the waveform observed for correct buttonpresses was not contaminated by mechanical or motor artifacts and due solely to the acoustic stimulation. The control waveforms for the four $\mathrm{Ss}$ not shown in Fig. 2 were similarly flat.

A complete set of AERs for one $S$ is given in Fig. 3 . In the experimental conditions (II, III, and IV) and particularly at the higher signal intensities of +2 and $+6 \mathrm{~dB} \mathrm{SL}$, a large positive peak is especially prominent

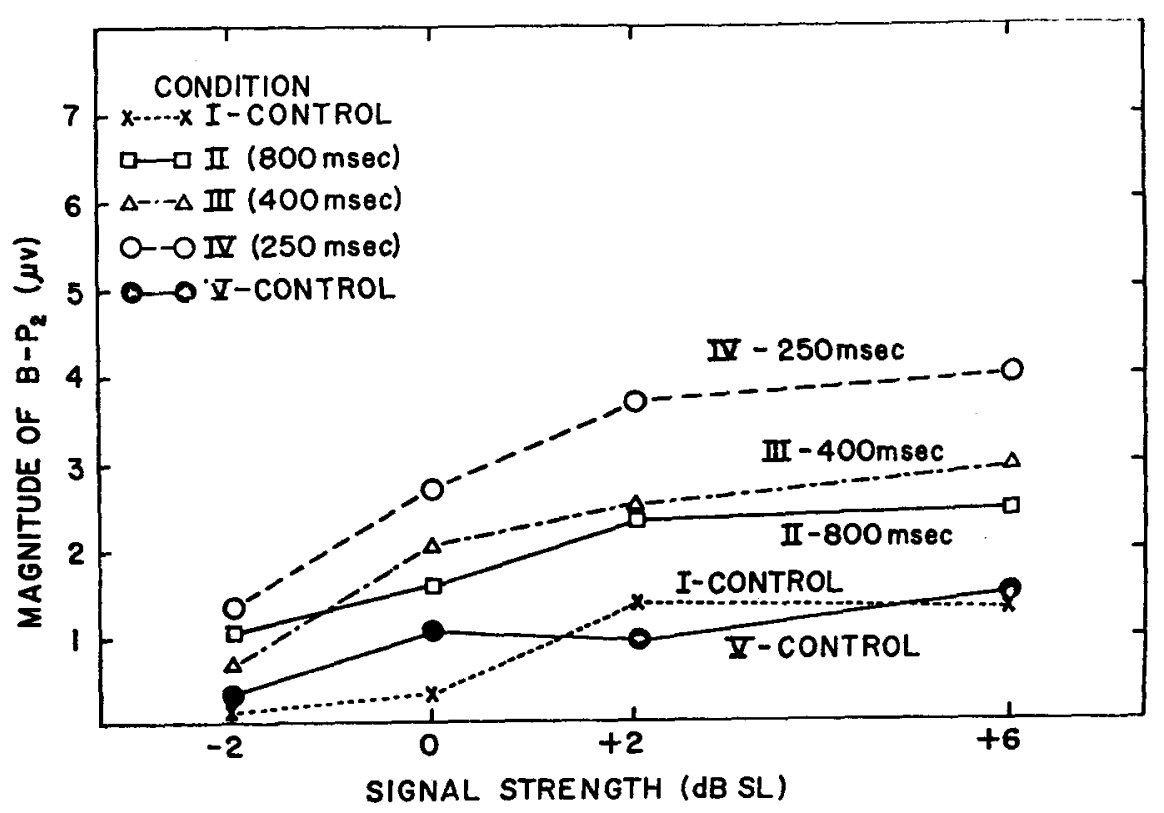

Fig. 4. Mean magnitude of $B-P_{2}$ for each condition as a function of signal strength. Data based on all seven Ss.

at approximately a 325-msec latency $\left(P_{2}\right)$. Although there was the expected variability of waveforms among $\mathrm{Ss}$, all Ss showed a clear $P_{2}$ to the +2 and $+6 \mathrm{~dB}$ SL stimuli in Conditions II, III, and IV. Because $P_{2}$ and the other major component of interest, $\mathrm{N}_{1}$, were not always conspicuous in the averaged AER, the temporal criteria described above were applied to all measurements of $N_{1}$ and $P_{2}$, even when the peak deflections happened to fall slightly outside the defined temporal intervals. The peak negative deflection did, in fact, occur outside the narrowly prescribed interval of 100-150 msec after stimulus onset $52 \%$ of the time. Nevertheless, in these cases, the waveform was still negative in the 100- to 150-msec interval. Thus, for $52 \%$ of the $N_{1}$ measurements, the values were submaximal. Peaks falling outside the temporal criterion were not confined to any particular experimental condition or signal strength but appeared to be distributed randomly among all combinations of condition and signal strength. Likewise, the peak positive deflection occurred outside its prescribed interval in $42 \%$ of the cases, thereby resulting in some submaximal measurements of $P_{2}$. These cases were also distributed among all the condition-signal strength combinations.

\section{$\mathbf{B}-\mathbf{P}_{2}$}

The mean magnitude of $\mathrm{B}-\mathrm{P}_{2}$ for each condition as a function of stimulus strength is plotted in Fig。 4. An analysis of variance indicated a significant effect for condition $(F=5.52, \mathrm{df}=4,24)$ and a significant interaction between condition and signal strength $(\mathrm{F}=22.94, \mathrm{df}=12,72)$. Dunnett's multiple comparison test for comparing means with a control (Winer, 1962) was used to identify which experimental conditions were significant for $P_{2}$. The test indicated that Experimental Conditions III and IV (CI: 400 and $250 \mathrm{msec}$, respectively) were significantly different from the mean of the combined control conditions, $I$ and $V$, at both +2 and $+6 \mathrm{~dB} \mathrm{SL}(\mathrm{p}<.05)$. Condition II (CI: $800 \mathrm{msec}$ ) did not differ significantly from the control mean at either signal strength. To test the hypothesis that $P_{2}$ increased with increasing stringency, i.e., reduction in the width of the CI, a nonparametric procedure was used. The nonparametric method used, the $L$ statistic (Page, 1963) assumes data of only ordinal strength and compares the rankings of the magnitude of $B-P_{2}$ according to experimental condition at a given signal strength. The test showed that the $\mathrm{B}-\mathrm{P}_{2}$ magnitudes increased with increasing task stringency at +2 and $+6 \mathrm{~dB} \mathrm{SL}$ $(\mathrm{p}<.05)$.

The Newman-Keuls procedure was used to compare the means for the +2 and $+6 \mathrm{~dB}$ SL signals for each of the experimental conditions to determine if $P_{2}$ increased with increasing signal strength. The test showed no significant differences for any of the conditions, indicating that over the range of $4 \mathrm{~dB}$ the $P_{2}$ component remained virtually unchanged.

\section{$\mathrm{B}-\mathrm{N}_{1}$}

As stated above, $\mathrm{N}_{1}$ was not observable in any of the responses at 


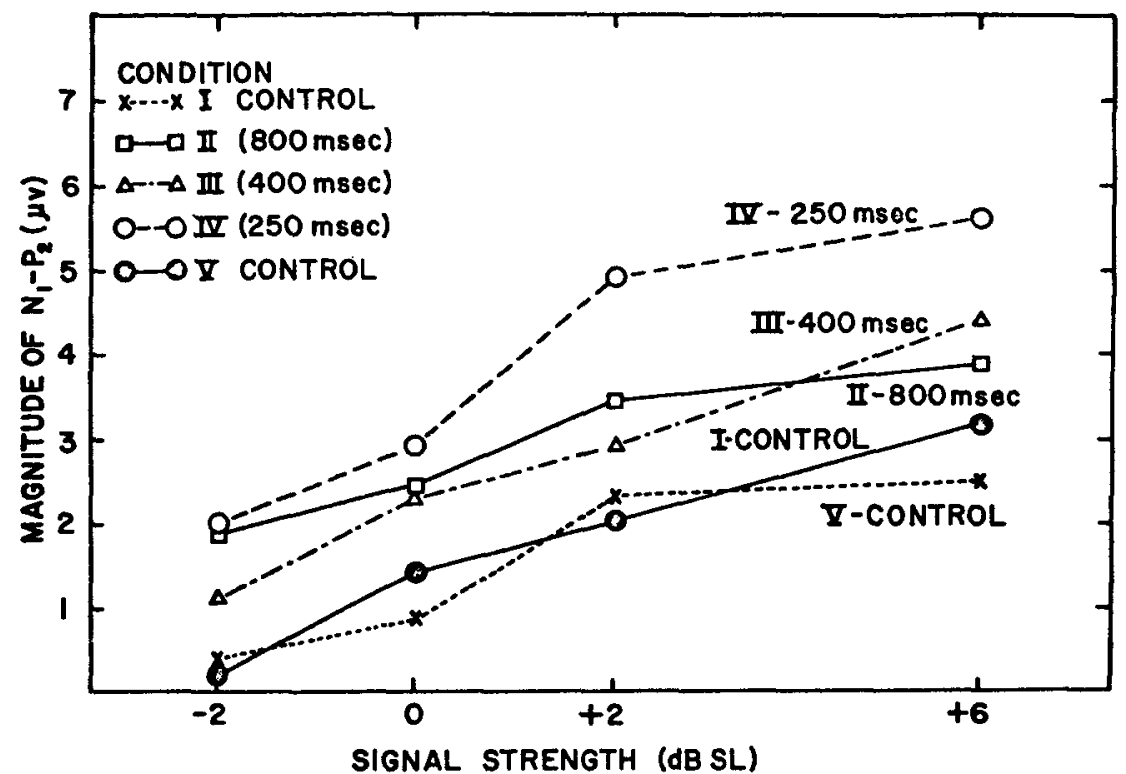

Fig. 5. Mean magnitude of $N_{1}-P_{2}$ for each condition as a function of signal strength. Data based on all seven Ss.

-2 or 0 dB SL. Consequently, analyses were made at only +2 and $+6 \mathrm{~dB}$ SL. The analysis of variance indicated that there was a significant effect for signal strength $(\mathrm{F}=8.49$, df $=1,6)$. Further analysis showed that only the differences between +2 and $+6 \mathrm{~dB}$ SL for Condition III (CI: $400 \mathrm{msec}$ ) were significant $(\mathrm{p}<.05)$. Nevertheless, similar trends of increased amplitude with increased signal strength were evident for the other experimental conditions. The analysis of variance also indicated that $\mathrm{N}_{\mathrm{s}}$ was not significantly different for any of the experimental or control conditions $(F=.60, d f=4,24)$. Therefore, the $\mathrm{B}-\mathrm{N}_{1}$ component of the AER was not influenced by task stringency to the degree that the $\mathrm{B}-\mathrm{P}_{2}$ component was affected. On the other hand, signal strength did appear to increase the amplitude of $B-N_{3}$ but not the amplitude of $\mathrm{B}-\mathrm{P}_{2}$.

\section{$N_{1}-P_{2}$}

The two components, B-P and $B-N_{1}$, were summed to yield the common peak-to-peak measure, $\mathbf{N}_{1}-\mathbf{P}_{2}$. The means of this measure appear in Fig. 5. Although the sum of the two components does not yield any new information, it does demonstrate how the overall peak-to-peak measure reflects the effects of two variables-stimulus strength and experimental condition. As would be expected from the previous analyses of the $P_{2}$ and $N_{1}$ components, an analysis of variance of the $N_{1}-P_{2}$ measurements shows that both signal strength $(\mathrm{F}=16.4$, df $=3,18)$ and condition ( $F=3.72$, df $=4,24)$ are significant effects.

\section{DISCUSSION}

The findings of this experiment tend to support the hypothesis that the $P_{2}$ component is a function of only the performance variable, at least within a narrow range of low signal levels. The amplitude of the $\mathbf{P}_{2}$ component correlated well with the performance criteria imposed upon Ss, so that the task which required maximal performance, i.e., bisecting the temporal interval with an accuracy of $\pm 125 \mathrm{msec}$, was associated with the largest $P_{2}$ component. As the difficulty of the task decreased, the magnitude of $P_{2}$ decreased. be exercised before excluding any influence of signal strength on the $P_{2}$ component, even though the effect of signal strength was statistically nonsignificant. Since it is extremely unlikely that either signal strength or performance criterion can be totally excluded when changes in $P_{2}$ or $N_{1}$ occur, the results would best be viewed in terms of the differential effects each variable has on $P_{2}$ and $N_{1}$.

By examining the differential effects of variables on $N_{1}$ and $P_{2}$, a finer analysis of the often-reported enhancement of the AER in attention paradigms may be possible. For example, Mast and Watson's (1968) conclusion that the increase in the AER due to attention is greater at low intensities than at high intensities might be qualified by stating that the $P_{2}$ increase due to attention is the same at both intensities. $N_{1}$, however, does increase with intensity but does not change as a result of attention. Therefore, the amount of change in the AER waveform due to the same Considerable caution, however, should levels of attention at high vs low intensities appears to be the sole result of the $N_{1}$ component.

Some evidence for the existence of independent components of the AER is provided by Vaughan and Ritter (1970) as mentioned above. They examined the AER waveform for different stimulus conditions and from different scalp electrode locations. The AER to periodic acoustic stimulation consisted primarily of two large deflections, the first a negative wave with a peak deflection at approximately $100 \mathrm{msec}$ and the second a positive wave at approximately $200 \mathrm{msec}$. Both waves were believed to originate in the primary auditory cortex. With aperiodic acoustic stimuli, however, a late positive component with a latency of $\mathbf{3 0 0}$ msec replaced the positive peak at $200 \mathrm{msec}$. This late positive component possessed a distribution over the scalp that was substantially different from the two earlier components that resulted from the periodic stimulation. The authors suggest that the generators for the late positive component are located over a wide portion of parietal-temporal association cortex.

Separate origins for the early components (less than 200-msec latency) and the late positive component would help explain why the late positive component, unlike the earlier components, does not change its latency as stimulus intensity changes. McCandless and Lentz (1968) have shown that the early components increase in latency as stimulus intensity decreases. The late positive component, however, does not appear to be affected by intensity. In the present study, the late positive component was located at approximately $300 \mathrm{msec}$ for $0,+2$, and $+6 \mathrm{~dB}$ SL stimuli. In other studies at higher intensities, e.g., $40 \mathrm{~dB}$ (Wilkinson \& Morlock, 1967), 75 dB (Sheatz \& Chapman, 1969), and $82 \mathrm{~dB}$ (Smith, Donchin, Cohen, \& Starr, 1970), the late positive component was still located at approximately 300 msec.

The presence or enhancement of a late positive component has often been attributed to an increase in the S's general activation level during the part of the experiment in which the $S$ is required to participate actively. Generalized arousal, however, would be expected to affect all stimuli in the experimental situation. In the present study, evoked responses to the "on" and "off" lights did not increase when the enhanced late positive component was present. On the contrary, the light-evoked responses usually decreased in magnitude throughout each experimental condition. 
Moreover, on the basis of previous experiments, generalized arousal does not present a convincing explanation. For example, Sheatz and Chapman (1969) presented relevant and nonrelevant stimuli in close temporal proximity, and still the differential enhancement occurred.

It has also been suggested that the late positive component may be the result of a motor response (Wilkinson \& Morlock, 1967). In the present experiment, the comparison of correct and incorrect responses eliminated this possibility by showing that the waveform was virtually flat following the buttonpresses and no subsequent acoustic stimulation (Fig. 2). Ritter and Vaughan (1969) have also verified that muscle potentials are not implicated in the presence of a late positive component.

Still another possible artifactual cause for the enhanced $P_{2}$ component reported here is the greater difference in the time between stimuli in the experimental conditions compared to the control conditions, Nelson and Lassman (1968) have shown that response amplitude is an increasing linear function of the logarithm of the intersignal interval (ISI). In the present experiment, the ISI was never less than 4 sec; the ISI was greater as the percentage of incorrect responses increased. Condition IV, with an average of $42 \%$ incorrect responses, would have average ISIs of less than 8 sec. According to Nelson and Lassman's data, the change in ISI from 4 to 8 sec would account for only a $10 \%$ increase in evoked response amplitude for a 60-dB SL signal. For a lower intensity, they would predict even less of a change. Since $\mathbf{P}_{\text {}}$ increased approximately 200\%-300\% in the experimental conditions, the ISI alone is not sufficient to account for the increase.

After this manuscript was submitted, a paper by Hillyard, Squires, Bauer and Lindsay (1971) appeared. Hillyard et al showed that a late positive component $\left(\mathrm{P}_{300}\right)$ occurred only for correct identifications (hits) for a low-intensity signal. Now, it is clear that $P_{3}$, is very probably identical to the deflection we refer to as $P_{2}$ in the present paper. Thus, with respect to terms appropriate to the detection paradigm as used by Hillyard et al, the data reported here might be representative only of the "hits." The misses are recovered only for illustrative purposes, and we have no firm basis on which to differentiate between the misses, while correct rejections could not occur in our procedure.

Thus, the present paper emphasizes the dissociation between the $N_{1}$ and $P_{30 \text { a components of the AER using }}$ one experimental procedure. Because the S's behavioral task was independent of signal strength, both $\mathrm{N}_{1}$ '(stimulus strength) and $\mathbf{P}_{3}$ o ("attention") could be examined for their independence. In the Hillyard et al paper and also in the more recent Picton, Hillyard, Galambos, and Schiff (1971) paper, the strength of the signal was not independent of the behavioral task and the data were not partitioned with respect to judgmental criterion. Thus, the relative effects of stimulus dimension and psychological set on the respective response components could not be separately assessed.

Although the experimental paradigm used here lacks the generality of the detection paradigm, it did allow the assessment of gradations in task difficulty and signal strength as independent variables. The data indicate that $P_{300}$ is graded with task difficulty and that $N_{1}$ is graded with signal strength.

\section{REFERENCES}

DAVIS, H, Enhancement of evoked cortical potentials in humans related to a task requiring a decision. Science, 1964, 145, 182-183.

GARCIA-AUSTT, E, BOGACZ, J", \& VANZULLI, A. Effects of attention upon yisual evoked responses Electroencephalography \& Clinical Neurophysiology, 1964, 17, 136-143.

GROSS, M. M. BEGLEITER, H. TOBIN M. \& KISSIN, B. Auditory evoked response comparison during counting clocks and reading, Electroencephalography \& Clinical. Neurophysiology, 1965 , $18,451-454$.

HAIDER, M., SPONG, R., \& LINDSLEY D. B. Attention vigilance, and cortical evoked potentials in humans. Science, 1964, 145, 180-182.

HILLYARD, S. A.. SQUIRES, K. C. BAUER, J. W., \& LINDSAY, P. H. Evoked potential correlates of auditory signal detection. Science, 1971, 172. signal detect

JASPER, $H$. H. The ten-twenty electrode system of the International Federation. Electroencephalography \& Clinical Neurophysiology, 1958, $10,371-375$.

MAST, T. E:, \& WATSON, C. S. Attention and auditory evoked response to low detectability signals. Perception \& Prychophysics, 1968, 4, 237-240.
McCANDLESS, G. A.. \& LENTZ, W. E. Amplitude and latency characteristics of the auditory evoked response at low sensation levels. Joumal of Auditory Research, 1968, 8, 273-282.

NELSON, D. A., \& LASSMAN, F. M Effects of intersignal interval on the human auditory evoked response. Journal of the Acoustical Society of America, $1968,44,1529-1532$.

PAGE, E. B. Ordered hypotheses for multiple treatments: A significance test for linear ranks. Journal of the American Statistical Association, 1963, 58, 216-230.

PICTON, T. W., HILLYARD, S. A., GALAMBOS, R., \& SCHIFF, M. Human auditory attention: $A$ central or peripheral process? Science, 1971, 173, 351-353.

RITTER, W., VAUGHAN, H. G., JR., \& COSTA, L. D. Orienting and habituation to auditory stimuli: A study of short term changes in average evoked responses. Electroencephalography \& CHnical Neurophysiology, 1968, 25, 550-556.

RITTER, W., \& VAUGHAN, H. G., JR. Average evoked responses in vigilance and discrimination: A reassessment Science, $1969,164,326-328$.

SATTERFIELD, J. H. Evoked cortical response enhancement and attention in man. A study of response to auditory and shock stimuli. Electroencephalography \& Clinical Neurophysiology, 1965, 19 , 470-475.

SHEATZ, G, C., \& CHAPMAN, R. M. Task relevance and auditory evoked responses. Electroencephalography \& Clinical Neurophy siology, $1969,26,468-475$.

SIFGEL, S. Nonparametric statistics for the behavioral sciences. New York: McGraw-Hill, 1956.

SMITH, D. B., DONCHIN, E., COHEN, L., \& STARR, A. Auditory averaged evoked potentials in man during selective bin aural listening. Electroencephalog. raphy \& Clinical Neurophysiology, 1970, 28, 146-152.

VAUGHAN, H. G., JR., \& RITTER, W. The sources of auditory evoked responses recorded from the human scalp. Electroencephalography \& Clinical Neurophysiology, 1970, 28, 360-367.

WILKINSON, R. HORLOCK, H. Auditory evoked response and reaction time. Electroencephalography \& Clinical Neurophysiology, 1967, 23, 50-56.

WILKINSON, R. T:, MORLOCK, H. C.. \& WLLIAMS, H. C. Evoked cortical response during vigilance. Psychonomic Science, 1966, 4, 221-222.

WINER, R. J. Statistical principles in experimental design. New York: MeGraw-Hill, 1962 .

\section{NOTE}

1. HAVOC (Histogrammer, Averager, and Ogive Calculator) is one of three similar special-purpose computers designed and built at Central Institute of the Deaf, St. Louis, Missouri. Briefly, the computer performs its own analog-digital conversion at a maximum rate of $10,000 \mathrm{~Hz}$; it subdivides the epoch chosen for averaging into 256 "bins" and quantizes the voltage of each bin in 256 steps. In addition to signal averaging, it can compute amplitude, latency, and interval histograms.

(Received for publication April 24, 1971.) 